\title{
Signal Monitoring by Spectrum Analyzers
}

\author{
Nabil Ali Sharaf Murshed ${ }^{1,}$ Abdelrasoul jabar Alzubaidi ${ }^{2}$ \\ ${ }^{1,2}$ Department of Electronic Engineering (Communication), ' ${ }^{1}$ Sudan Academy of Sciences, \\ ${ }^{2}$ Sudan University of Science and Technology
}

\begin{abstract}
Signal monitoring systems can range from extremely simple to very complex fixed-site-systems. This paper will review some of the basic components that can be used to assemble a signal monitoring system. The most basic of signal monitoring systems could simply be a radio and an antenna. To learn more about the signal it is necessary to measure frequency and amplitude of the signal. These requirements suggest that the spectrum analyzer can be the foundation of many signal monitoring systems.
\end{abstract}

Keywords: - Signal monitoring systems, antenna, and spectrum analyzer.

\section{INTRODUCTION}

Signal being transmitted, receiver and travel at least significant portion of their path in free space this clearly an essential characteristic in certain application such as mobile phone, satellite communication, broadcasting and radio navigation.

In other application such as fixed links and fixed wireless (FWA), it provides a key advantage the avoidance of upfront cost of laying transmission lines.

The main disadvantage however is that transmission over free space means that stray signals from one radio communication system can easily interfere with the proper reception of another. Interference is unavoidable and ever present.

The impact of excessive interference ranges from simple inconvenience to individual users to, on occasions, the undermining of the viability of networks suffering interference. At the very extreme, it can have safety of life implications, for example where radio systems used by the emergency services suffer interference. Furthermore radio signals do not respect national borders. Thus, if not properly managed, signals emanating from one country can unduly interference with system in other countries.

This propensity to interfere, locally and regionally, is the key factor rendering the radio spectrum a resource. National regulatory authorities (NRAs) throughout the world have therefore regarded it as one of their central duties to ensure both an acceptable interference environment as well as maximizing the (technically) efficient use of the available spectrum. This has resulted in implication of international regulation and technical considerations on market mechanisms in spectrum management, a significantly regulated environment in radio communications, with the vast majority of NRAs in the world retaining close control on deciding both the type of permitted services and which organizations should be licensed to operate in a given trencher of spectrum. So, we need radio monitoring system that can manage radio efficiently and measure radio quality accurately through spectrum analysis for protecting wireless equipment and maintaining quality level of radio, communication service. Also, since conventional radio monitoring system can't measure frequency efficient use investigation and spectrum analysis that is equivalent to occupied bandwidth measurement, broadband frequency measurement, high-speed spectrum measurement, unwanted electromagnetic signal in radio quality measurement, radio monitoring system need to be developed for executing efficient radio monitoring work with reservation measurement function and automatic result storage function that can be done accurate radio measurement of local operators.

\section{II.}

\section{SIGNAL MONITORING GOALS}

- Frequency management:

- Monitor RF bands to determine compliance with local laws and regulations

- Monitor assigned frequencies to look for interfering signals or to determine if your transmitters are working properly

- Manage RF assets on large industrial/government sites, like airports, missile launch facilities, and test ranges

- Look for RF sources that might be causing out of band interference to other equipment such as medical equipment

- Perform site surveys prior to installation of a repeater or cellular base station.

- Signal surveillance( monitoring):- 
- Law enforcement or other government agencies could monitor other people's transmissions to extract intelligence

- Sweep rooms or buildings for bugs or other RF emitters

Basic Equipment

- For the most basic spectrum monitoring tasks a receiver and antenna are required.

- Suggested equipment could include:

- Spectrum Analyzer (receiver)

- Antenna

- Antenna transmission lines

- Preamplifier

- Specialized software

\section{1-The receiver}

Receiver is really the heart of the signal monitoring system. As mentioned previously, signal monitoring systems can range from simple to complex. For general signal monitoring requirements, the spectrum analyzer is a very attractive receiver choice. The spectrum analyzer's extremely broad frequency coverage and excellent dynamic range make it a logical building block for signal monitoring systems. In some applications that require $100 \%$ probability of intercept (POI) the swept tuned spectrum analyzer may not be the best tool. In these cases specialized receiving equipment may be required. However, for narrow spans, less than $10 \mathrm{MHz}$, the Agilent PSA series spectrum analyzer can be used in FFT mode to improve POI

\section{2-PSA Series Spectrum Analyzer}

The PSA series spectrum analyzers are well suited for signal monitoring applications.

The PSA is offered in five models covering the following frequency ranges: $3 \mathrm{~Hz}$ to $6.7 \mathrm{GHz}, 3 \mathrm{~Hz}$ to $13.2 \mathrm{GHz}$, $3 \mathrm{~Hz}$ to $26.5 \mathrm{GHz}, 3 \mathrm{~Hz}$ to $44 \mathrm{GHz}$, and $3 \mathrm{~Hz}$ to $50 \mathrm{GHz}$. The PSA family is unique, in that it incorporates a revolutionary $100 \%$ digital IF. All of the traditional analog filters and IF signal processing have been replaced with a custom ASIC that performs these through DSP.

The PSA's digital IF provides improved IF filtering and greatly improved measurement accuracy. In addition, the PSA's DSP provides a host of detector choices to aid signal characterization. The PSA family features many single button measurements to simplify modern RF measurements. Some of these measurements that are of interest in a signal monitoring environment are occupied bandwidth, integrated channel power, adjacent channel power, spectrum masks.

The PSA also provides an amplitude correction function. Amplitude correction allows the user to specify amplitude correction table to apply to measurement data. In a signal analysis role amplitude correction can be used to correct measured data for antenna gain, transmission line losses, and preamplifier gain. In addition to amplitude correction, the PSA includes a set of measurement limit lines. The limit lines can be programmed to generate an alarm when a signal's amplitude exceeds the limit setting in a specific frequency range. Following our discussion of antennas and preamplifiers we will discuss the use of limit lines and amplitude correction.

\section{3-Antennas}

- The following antenna parameters must be considered when selecting an antenna for signal monitoring:

- Gain or antenna factor

- Beam width

- Polarization

- Impedance and match

- Frequency range or bandwidth

- Power handling capability

- Size and weight

Many factors will influence your choice of antennas. Generally, most signal monitoring applications require portable operation, which limits the practical size and weight of antennas. However, if very small signals must be analyzed larger antennas will be required. Antenna polarization is another important consideration. If the polarization of received signal is unknown a circular polarized antenna may be the best choice, since it works equally well for both horizontal and vertical polarization.

Line of bearing of the measured signal may be determined by various techniques.

One simple method is to use a spinning receive antenna. By correlating the signal with the direction the antenna was pointed when the signal was received the signal's line of bearing may be determined.

Another very important antenna parameter is bandwidth. If you are only looking over a narrow communications band, simple narrow-band antennas will provide an economical solution. However, if you must survey a broad 
frequency spectrum a broadband antenna will be required. Associated with bandwidth is dispersion. Some antennas, while broadband will induce different time delays to different frequencies.

These changes in delay can greatly distort very short pulses.

Generally, antennas designed for Electromagnetic interference (EMI) and electromagnetic compatibility (EMC) applications work very well for signal monitoring applications.

\section{Antenna Gain}

- Several factors relate the antenna's output voltage or power to incident field strength and power density. The antenna gain $\mathrm{G}$, effective area Ae, antenna factor AF, and beamwidth can be related mathematically.

$$
\begin{aligned}
& G=\eta G_{D} \quad G_{D}=\frac{4 \pi}{\theta \phi(\text { radians })^{2}}=\frac{41,253}{\theta \phi(\mathrm{deg})^{2}} \\
& A_{e}=\frac{G \lambda^{2}}{4 \pi}
\end{aligned}
$$

The directive gain of an antenna is a measure of its ability to concentrate energy in one direction. Mathematically, directive gain can be defined as the antenna maximum radiation intensity divided by its average radiation intensity.

Since theaverage radiation intensity over a solid angle of $4 \pi$ radians is equal to the total power

$$
G_{\mathrm{D}}=\frac{4 \pi(\text { maximum power radiated/unit solid angle })}{\text { total nower radiated }}=\frac{4 \pi}{B}
$$

Radiated divided by $4 \pi$, directive gain can be expressed as:

Where B is the beam area of the antenna, this is the solid angle through which all of the antenna's power passes. If $\theta$ and $\varphi$ are the half power beam width in two orthogonal planes then B is approximately equal to $\theta \varphi$. This results in a directive gain of:

$$
G_{D}=\frac{4 \pi}{\theta \phi(\text { radians })^{2}}=\frac{41,253}{\theta \phi(\text { deg })^{2}}
$$

Since our antenna is non ideal, its actual power gain will be somewhat less than its directive gain. The ratio of power gain $(\mathrm{G})$ divided by directive gain $(\mathrm{G} D)$ is the antenna efficiency factor $\eta$. Another useful antenna parameter related to gain is the effective aperture or area Ae presented by the antenna to the incident wave. The gain $\mathrm{G}$ and effective area of a loss less antenna are related by:

$A_{e}=\frac{G \lambda^{2}}{4 \pi}$

\section{Determining Field Strength}

- The antenna's measured output signal power can be used to determine the power density of the incident wave that is intercepted by the antenna.

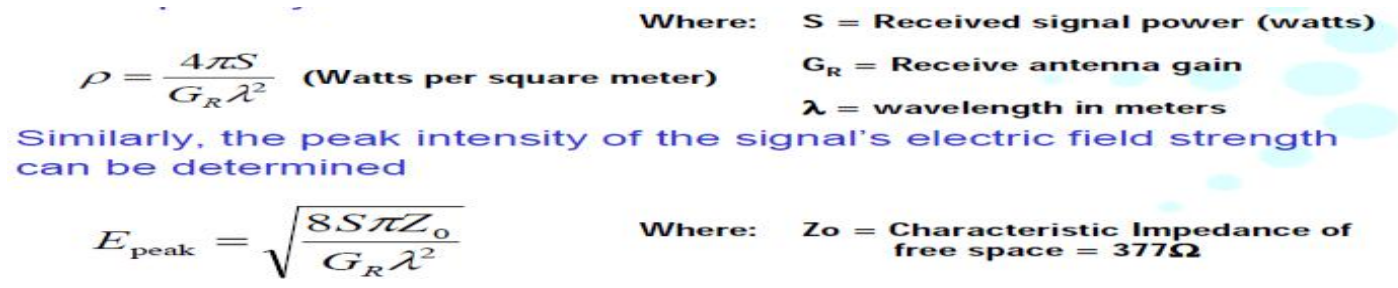

The antenna's output signal power (S) is simply the product of the antenna's effective aperture Ae multiplied by the power density of the incident wave. Assuming the signal's source is an isotropic radiator and multipath and fading are not considered, the free space power density will be the source's output power divided by the surface area of a sphere with radius $\mathrm{R}$

\section{PREAMPLIFIERS}

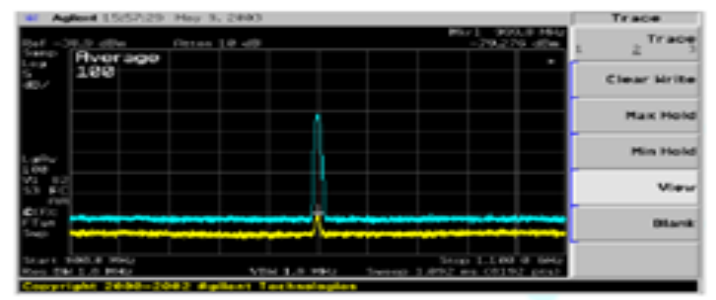


A preamplifier can be used to improve the spectrum analyzer's sensitivity.

The sensitivity of a spectrum analyzer is specified as displayed average noise level (DANL). For signal monitoring applications the smallest signal that can be displayed is one equal in amplitude to DANL. Another common way to specify the sensitivity of a receiver is by its noise figure F. Noise figure can be defined as the ratio of output to input signal to noise ratio or $\mathrm{F}=(\mathrm{Si} / \mathrm{Ni}) /(\mathrm{So} / \mathrm{No})$, where $\mathrm{Si}$ and $\mathrm{Ni}$ are the input signal and noise and So are the output signal and noise. In the special case of a spectrum analyzer, the output signal is equal to the input signal times the gain of the analyzer, which is unity because the output signal on the display is the same amplitude as the input level at the RF connector. Therefore, the expression for noise figure can be simplified to $\mathrm{F}=\mathrm{No} / \mathrm{Ni}$. To determine $\mathrm{F}$ we simply divide the DANL by the available noise power at the input, or by KTB. Expressed in $\mathrm{dB}$ the resulting noise figure would be:

$\mathrm{F}(\mathrm{dB})=10 * \log (\mathrm{F})=\mathrm{DANL}-(-174 \mathrm{dBm} / \mathrm{Hz})-10 * \log (\mathrm{B})$ For the lower trace we see that $\mathrm{FSA}=-83.9 \mathrm{dBm}+174-62.6=27.5 \mathrm{~dB}$.

A noise figure of $27.5 \mathrm{~dB}$ is pretty good for a spectrum analyzer with $10 \mathrm{~dB}$ input attenuation, but somewhat poor for a signal monitoring receiver. The upper trace shows the same signal after being amplified by a preamplifier with a gain of $25 \mathrm{~dB}$ and a noise figure of $7.77 \mathrm{~dB}$. The new DANL with the preamplifier is:

DANL2 $=$ KTBFeqG2 $-2.5 \mathrm{~dB}=-174 \mathrm{dBm}+62.6+8.9+25-2.5=-80 \mathrm{dBm}$ Where Feq is the system noise figure of the analyzer and preamp combined and the $2.5 \mathrm{~dB}$ term is a correction term for noise signals (see Agilent Application Note 150).

\section{AMPLITUDE CORRECTIONS}

- Measured signal amplitudes can be corrected

for:

- Antenna gain

- Transmission line loss

- Preamplifier gain.

- Four separate correction tables are provided

- Correction data can be entered manually or downloaded from a computer

- Correction tables can be stored with instrument tates

- Corrections can be turned on or off at will.

Amplitude correction is essentially a necessary feature for most signal monitoring applications. Generally, not only is the frequency and bandwidth of a signal important, but the amplitude of the measured signal is also important. The PSA series spectrum analyzers provide four amplitude correction tables that are defined as:

Antenna, cable, other, and user. Each of these corrections can be turned on or off independently of the others. In addition a separate key labeled "Apply Corrections" must be pressed to invoke the corrections. Antenna, cable, other, and user corrections are generally entered as positive values, which indicate a loss in the external device. Negative values would be used to represent gain in the external device. Correction tables can be entered manually from the front panel of the spectrum analyzer or they can be downloaded from an external computer.

Correction factors can also be used to provide more complex corrections to measured data.

For example, if the user desires to correct screen data to free space power density a suitable correction table can be calculated based on antenna gain and frequency. Modifying our equation for power density on Slide 10 to replace wavelength with frequency yields a correction factor in $\mathrm{dB}$ of:

$10 * \log (4 \pi)-20 * \log (\mathrm{c})+20 \log (\mathrm{F})-\mathrm{GR}=20 \log (\mathrm{F})-\mathrm{GR}-159 \mathrm{~dB}$

Assuming we are measuring a signal at $2.4 \mathrm{GHz}$ with an antenna whose gain is $10 \mathrm{~dB}$, we would apply a correction factor of $18.6 \mathrm{~dB}$. If we leave the spectrum analyzer in its preset mode, of displaying power in $\mathrm{dBm}$, our display would now be calibrated for $\mathrm{dBm} / \mathrm{m} 2$, for this specific antenna. By changing the amplitude display to display in Watts we would be able to directly read power density in Watts/ $\mathrm{m} 2$.

\section{CONCLUSION}

- Commercially available spectrum analyzers are an excellent foundation for signal monitoring applications such as frequency management and signal surveillance

- Recent enhancements to the Agilent PSA series of spectrum analyzers, coupled with appropriate accessories, can provide excellent capability and flexibility to system designers, integrators, and users

- Additional Agilent products contribute to a robust signal monitoring solution

- 89601A VSA software

- Web Remote software 
- E8257C PSG signal generator

- 5485xA Infinities series oscilloscope

\section{REFERENCES}

[1] R, S, Spectrum Analyzers vs. Monitoring Receivers,Paul Denisowski, Application Engineer Rohde \& Schwarz

[2] Thomas Petruzzellis, 22 Radio Receiver Projects for the Evil Genius, Copyright @ 2008 by The McGrawHill Companies, Inc.

[3] Radio Spectrum Management Module 5 of ICT Regulation Toolkit McLean Foster \& Co. in collaboration Martin Cave and Robert W. Jones

[4] LS Summit 2013 - Automatic Radio Monitoring Hochschule Offenburg University of Applied Sciences 